\title{
DRESS syndrome associated with sulfasalazine therapy.
}

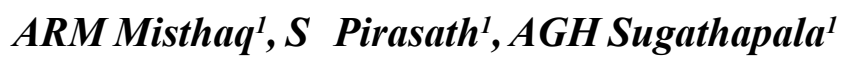

${ }^{1}$ Colombo South Teaching Hospital, Kalubowila, Sri Lanka.

\begin{abstract}
The Drug Reaction with Eosinophilia and Systemic Symptoms (DRESS) is a rare life threatening drug hypersensitivity reaction which include a skin rash, fever, lymphadenopathy, hematologic abnormalities and internal organ involvement. Here, we reported DRESS syndrome case in a 55 year old female patient following intake of sulfasalazine for her knee joint pain who was completely recovered with appropriate management.
\end{abstract}

\section{Key words}

DRESS Syndrome, Sulfasalazine, Liver enzymes, Maculopapular rash

\section{Introduction}

DRESS syndrome is a severe drug induced reaction which is manifested as skin rash, fever, hematological abnormalities and internal organ involvement (1). Here, we reported a case of DRESS syndrome in woman who fulfilled the most of the diagnostic criteria of DRESS syndrome established by a Japanese consensus group.

\section{Case report}

A 55 year old patient with ischaemic heart disease, hypertension and dyslipidemia on regular clinic follow up. She presented with two day history of generalized itchy rash first appeared on the face then spread to other part which was associated with fever and facial swelling. She also complained of difficulty in breathing. She has no history of abdominal pain. On further inquiry, she gave a history of intermittent right knee joint pain for 7 years suggestive of mechanical type. One month back she met a GP with worsening of right knee joint pain for last 2-3 months who has prescribed sulfasalazine and some painkiller. She denied history of allergic reaction to any drugs or food in the past. On examination, she was febrile and there was generalized maculopapular rash sparing upper limbs and mucous membrane. There were few posterior cervical lymph nodes palpated. Her pulse rate was $92 \mathrm{bpm}$ and blood pressure was 140/90mmHg. Rest of systemic examinations were unremarkable.Her full blood count showed leukocytosis $(19.81 \times 103)$ (Normal range $(4 \times 103$ - 11×103) without eosinophilia (14.4\%) (Normal range $(0 \%-6 \%)$. Her inflammatory markers were normal (ESR22 mm/1st hour, CRP-32 mg/L), liver enzymes were elevated (AST 82 U/1, ALT 139 U/l) and renal function tests were normal. Septic screening was negative. An ultra sound scan of the abdomen was performed which revealed no hepatosplenomegaly. According to diagnostic criteria of DRESS syndrome established by a Japanese consensus group, she was fulfilled six of the criteria to diagnosis of DRESS including fever, maculopapular skin eruption, lymphadenopathy, eosinophilia, atypical lymphocytes and abnormal liver function. sulfasalazine was identified as an offending drug in our patient and was withdrawn it and corticosteroid therapy was commenced . The skin eruption was treated with topical steroid and emollient with monitoring her electrolytes, hydrated adequately. Her skin lesion was healed and eosinophil count and liver enzyme level have come to baseline. She was fully recovered from DRESS syndrome and being followed up in the clinic. Her oral prednisolone was tapered off over 4 weeks duration.

\section{Discussion}

DRESS syndrome is rare drug induced hypersensitivity reaction and is manifested a skin rash, fever, lymphadenopathy, hematological abnormalities and systemic involvement (1). 
The term 'drug rash with eosinophilia and systemic symptoms' was coined by Bocquet (2). The common drugs causing DRESS syndrome includes allopurinol, carbamazepine, lamotrigene, phenytoin, sulfasalazine, minocycline, dapsone, sulfamethoxazole $(3,4)$. The pathogenesis of DRESS syndrome is not well understood. But a genetic component that alters immune response, a triggering factor such as a viral infection and a defect drug metabolism result in failure to eliminate drug intermediates are essential components (5). HLA is well described with drug hypersensitivity can be associated with DRESS syndrome. An accumulation of toxic metabolites due to genetic deficient in drug metabolism can cause cell death or inducing secondary immunological phenomena as result of eosinophilic activation and potentiation of inflammatory cascade. It usually appears 2 to 8 weeks after administration of the causative drug and the symptoms may persist or aggravate despite the discontinuation of causative drug. Our patient developed DRESS syndrome one month after the commencement of sulfasalazine therapy. The skin manifestations varies in form of an urticarial, maculopapular rash and sometimes vesicles, bullae, pustules and purpura. Mucocutaneous involvement is not seen in DRESS syndrome making it distinguish from other sever drug eruptions such as Steven-Johnson syndrome (7). The visceral involvement include hepatitis, pneumonitis, myocarditis, pericarditis, nephritis, and colitis. The most commonly found hematological abnormality is eosinophilia (6). The onset of eosinophilia may get delayed up to 1-2 weeks and even up to the time where elevations in liver enzyme levels return to baseline (7). The eosinophilia or lymphocytosis (40\%) are commonly seen with DRESS syndrome (8). According to the Diagnostic criteria for DRESS syndrome published by a Japanese Consensus group, she has fulfilled the diagnosis of DRESS syndrome. The main treatment of DRESS syndrome is withdrawal of offending drug, corticosteroids and supportive treatment (7). The outcome is better if the corticosteroids are started at the early stage (9). In our patient, we identified possible offending drug and withdrawn it and treated with both topical and systemic corticosteroids and supportive care.

The clinicians should think about possibility of DRESS syndrome in presence of diagnostic criteria for drug-induced hypersensitivity syndrome in suspicious cases following drug therapy. It will be helpful to discontinue the drug and to start appropriate actions to prevent consequences of it.

\section{Informed consent}

The informed written consent was obtained from patient for publication of case report.

\section{References}

1. Chaiken B, Goldberg B, Segal J. Dilantin hypersensitivity: report of a case of hepatitis with jaundice, pyrexia, and exfoliative dermatitis. NEngl J Med. 1950; 242:897-8.

2. Bocquet H, Bagot M, Roujeau JC. Drug-induced pseudolymphoma and drug hypersensitivity syndrome (Drug Rash with Eosinophilia and Systemic Symptoms: DRESS). Sem Cutan Med Surg. 1996; 1:250-7.

3. Tennis P, Stern RS. Risk of serious cutaneous disorders after initiation of use of phenytoin, carbamazepine, or sodium valproate: a record linkage study. Neurology. 1997;49:542-546.

4. Peyriere H, Dereure O, Breton $\mathrm{H}$, et al. Variability in the clinical pattern of cutaneous side-effects of drugs with systemic symptoms: does a DRESS syndrome really exist? Br J Dermatol. 2006;155:422-428.

5. Roujeau JC, Stern RS. Severe adverse cutaneous reactions to drugs. $N$ Engl J Med. 1994;331:1272-1285

6. S. H. Kardaun, P. Sekula, L. Valeyrie-Allanore et al., "Drug reaction with eosinophilia and systemic symptoms (DRESS): an original multisystem adverse drug reaction. Results from the prospective RegiSCAR study," British Journal of Dermatology.2013; 169(5): 1071.

7. Sonal Choudhary, MD, Michael McLeod, MS, Daniele Torchia, MD, and Paolo Romanelli, MD. Drug Reaction with Eosinophilia and Systemic Symptoms (DRESS) Syndrome. $J$ Clin Aesthet Dermatol. 2013; 6(6):31-37

8. Callot V, Roujeau JC, Bagot M, et al. Druginduced pseudolymphoma and hypersensitivity syndrome. Two different clinical entities. Arch Dermatol. 1996;132:1315-1321

9. Santiago F, Goncalo M, Vieira R, et al. Epicutaneous patch testing in drug hypersensitivity syndrome (DRESS) Contact Dermatitis. 2010;62:47-53. 